OAI-PMH: http://www.indteca.com/ojs/index.php/Revista Scientific/oai

Artículo Original / Original Article

\title{
La Educación de la Mancomunidad Indígena Timotes
}

Autora: María del Carmen Pérez Paredes Universidad Fermín Toro, UFT

miduam@hotmail.com

Mérida, Venezuela

https://orcid.org/0000-0002-5754-8507

\section{Resumen}

La presente investigación tiene como propósito en reconstruir el modelo pedagógico subyacente en el proceso de la educación étnica que practica la Mancomunidad Indígena El Paramito, la problemática se presenta en la actual Etnia Timotes, donde paulatinamente se está perdiendo la lengua originaria, su propia cultura, tradiciones, mitologías, costumbres y sus formas de organización mancomunada. El marco teórico se orientó en función de la Educación Intercultural, Yampara (2010) y modelos pedagógicos según Flórez (2008). Metodológicamente se utiliza el paradigma cualitativo, se asume el método etnográfico dado que es el que permite aprehender la manera de como un grupo social se relaciona con los demás, se aplica como técnica de recolección de datos las siguientes: observación directa y entrevista en profundidad. Para dar respuesta a los valores educativos entre los cuales están: aprender haciendo, imitación, respeto y solidaridad, los culturales hallados en la comunidad fueron: superstición, temor a la naturaleza, medicina tradicional, ritual y creencias. Por otro lado, al aplicar las entrevistas, se resaltan las categorías y subcategorías sobre la cultura de la Mancomunidad indígena El Paramito. Además, reformar la forma de educar perteneciente a la etnia como esencia a investigación. Finalmente se concluyó en sensibilizarlos a su empoderamiento de su cultura, formas de vida, idioma, su saber étnico, credos, para defender y proteger sus derechos como grupo originario.

Palabras clave: educación intercultural; modelo educacional; lengua en vía de desaparición. 


\section{The education of the Commonwealth indigenous Timotes}

\begin{tabular}{|c|}
\hline $\begin{array}{l}\text { Abstract } \\
\text { The purpose of this research is to reconstruct the pedagogical model } \\
\text { underlying the process of ethnic education practiced by the El Paramito } \\
\text { Indigenous Commonwealth, the problem is presented in the current Timotes } \\
\text { ethnic group, where the original language, its own culture, is gradually being } \\
\text { lost, traditions, mythologies, customs and their forms of joint organization. The } \\
\text { theoretical framework was oriented in terms of Intercultural Education, } \\
\text { Yampara (2010) and pedagogical models according to Flórez (2008). } \\
\text { Methodologically, the qualitative paradigm is used, the ethnographic method is } \\
\text { assumed given that it is the one that allows us to apprehend the way in which } \\
\text { a social group relates to others, the following are applied as a data collection } \\
\text { technique: direct observation and in-depth interview. To respond to the } \\
\text { educational values among which are: learning by doing, imitation, respect and } \\
\text { solidarity, the cultural findings found in the community were: superstition, fear } \\
\text { of nature, traditional medicine, ritual and beliefs. On the other hand, when } \\
\text { applying the interviews, the categories and subcategories on the culture of the } \\
\text { El Paramito indigenous community are highlighted. In addition, to reform the } \\
\text { way of educating pertaining to the ethnic group like essence to investigation. } \\
\text { Finally, it was concluded to sensitize them to their empowerment of their } \\
\text { culture, ways of life, language, ethnic knowledge, creeds, to defend and protect } \\
\text { their rights as an original group. }\end{array}$ \\
\hline
\end{tabular}

Date Received: 10-08-2018

Date Acceptance: 01-11-2018 


\section{Introducción}

La presente investigación tiene como objetivo develar los valores educativos y culturales de la mancomunidad indígena en su actividad cotidiana, tomando en cuenta que somos un país con una gran diversidad cultural y, que para construir una identidad cultural sólida, hay que tener siempre presente las raíces de identidad como venezolanos, haciendo conciencia de los símbolos propios: étnicos, lengua, tradiciones, costumbres, religión e historia, fortaleciendo cada día las fuerzas de identidad plural en la de unidad nacional. En este sentido es pertinente señalar que los pueblos indígenas cuentan con leyes donde el estado les garantiza sus derechos consagrados en las mismas, afirmando la participación en la sociedad para la protección de su cultura y derecho a una educación adecuada de los pueblos y comunidades indígenas basada en medios de socialización mediante los cuales se transmiten y modernizan los elementos de su cultura a pesar de la protección legal y del establecimiento de una educación intercultural la cultura indígena poco a poco se ha ido extinguiendo con el transcurrir del tiempo en virtud al dinamismo y complejidad de los fenómenos étnicos y el intercambio permanente con otras culturas, tal es el caso del grupo de los Timotes, donde paulatinamente se está perdiendo la lengua originaria adoptando la segunda lengua el castellano, su propia cultura, tradiciones mitologías costumbres y su formas de organización mancomunada.

Las comunidades indígenas han estado sujetas a confiscación y explotación de su identidad cultural por organismos de sociedades distintas a las de sus generaciones. Es indispensable resguardar su herencia sobre su hábitat, su propiedad intelectual, lengua, tradiciones, costumbres, modos de vida y su territorio.

Para tal efecto es necesario brindar a estas comunidades una adecuada orientación y formación para que participen activamente en la conservación de su patrimonio cultural, con énfasis en la integración de sus patrimonios 
sensibles y sutiles, que no es otra cosa que su propia vida o espacio geográfico.

En vista, de una larga historia los saberes étnicos han pasado inadvertidos, han sido limitados o considerados como aspectos externos, por su inconstante transmisión oral o falta de sistematización el propósito es reconstruir un modelo pedagógico subyacente acerca de la transmisión de la educación étnica en el seno de la mancomunidad indígena Timotes, con la finalidad de evitar la pérdida del legado cultural de esta etnia.

Se considera entonces, la importancia del conocimiento y creencia en una diversidad educativa y su complemento primordial para alcanzar el respeto efectivo de las culturas étnicas que constituyen en su conjunto la identidad nacional y, como parte de esa diversidad es preciso que se les otorgue en el aspecto educativo, económico, social y cultural la oportunidad de participar de manera equitativa en cada población indígena. Según la localización geográfica de la Mancomunidad Indígena El Paramito o Pueblo Indígena Timotes, está en el sector Paramito Alto, jurisdicción de la parroquia capital Timotes, municipio Miranda, Estado Mérida. Limitando por el Norte con la Quebrada de Bailón, por el Sur con la Quebrada Chamarú, por el Este con El Paramito y por el Oeste con los Altos de Miranda.

Se encuentra ubicada aproximadamente a ocho kilómetros del casco central del pueblo de Timotes, en el estado Mérida. Presenta un clima cuya temperatura es de 12 grados promedio anual, cuando hace frío y hasta 18 grados en época de verano. La vegetación abundante es el frailejón, cuenta con terrenos fértiles para la agricultura, cuyos rubros principales son: papa, zanahoria, coliflor, repollo, alcachofa, ajo porro, cilantro, perejil, lechuga, cebolla y alguna variedad de flores específicamente rosas (Bastidas, 2003, pág. 7).

Además de describir la historia y cultura indígena, por nuestros antepasados que la han escrito con grandeza y abnegación, sus propios hijos, desde el aborigen Timotes hasta nuestros actuales que día a día consolidan 
este hermoso terruño.

Hoy día, Timotes percibe grandes cambios como pueblo, los cuales marcan el proceso de transformaciones diversas que ha beneficiado a nuestra gente. Así en estos parajes, comienza a escribirse hermosas páginas para la historia.

En la actualidad la agrupación es ocupada por sus habitantes de la comunidad Timotes, admiten la posesión y pertenencia de la agrupación étnica para alcanzar y conservar, transferir sus culturas a diferentes concepciones. El grupo étnico Timotes goza de una serie de normas por las cuales se guían oralmente dentro de la agrupación. Estas normas rigen la conducta para estar dentro de la etnia y la manera de desenvolverse dentro de la comunidad de El Paramito. Según algunos estudios, los sucesores de esta tribu en tiempos pasados se resistieron a la partición de sus tierras negándose a la donación de cada uno, solicitando la permanencia del grupo de manera mancomunada y la asignación por el medidor de la comunidad indígena Timotes.

\section{Bases Teóricas}

\subsection{Educación Intercultural}

La educación intercultural, es un enfoque educativo actual que contiene una visión holística de carácter inclusivo, con otra perspectiva de entender y vivir la educación a través de la diversidad cultural:

Interculturalidad es el medio que orienta a forjar el respeto mutuo, poner en la balanza de la historia los valores éticos y ecológicamente compatibles y que históricamente, nos han dejado como herencia a través de una riqueza extraordinaria de elementos (Pérez, 2016, pág. 171).

Asimismo, la educación intercultural y empoderamiento de las diferencias y diversidad educativa que enriquece y nutre en el contexto socialeducativo. En este orden de ideas la: 
Interculturalidad es, concertar dialogar -entre pueblos - entre los distintos modos de "saber hacer", los conocimientos, la sabiduría de las diversas civilizaciones en condiciones de equidad, respetando los derechos constituidos y la cultura. Es decir, compartir, complementar, intercambiar y reciprocar los saberes y valores de estos pueblos por medio de métodos concibiendo el respeto de su identificación y dignidad cultural (Yampara, 2010, pág. 27).

Si bien es cierto, la diversidad cultural accede a un aprendizaje y a un estándar formativo que permite un conjunto de dogmas, creencias, cultura, tradiciones, costumbres, donde estos pueblos exploran su acervo cultural reconociendo toda la desigualdad de esta población indígena.

Dentro de este contexto en nuestro país, se comienza a hablar de la diversidad cultural desde la antigüedad, refiriéndose a la enseñanza donde prevalezca una formación conforme al acervo cultural y la armonía de las líneas políticas de todos sus órganos. Bajo este propósito, se pretende el desarrollo del pensamiento crítico y creativo, así como las capacidades intelectuales de las sociedades en igualdad de condiciones, por lo cual algunas organizaciones declaran el "Año Internacional de la Juventud" en ocasión de dirigir la atención hacia tres temas vinculados con la interculturalidad: la participación, el desarrollo y la paz.

De esta manera, se pretende implementar un modelo pedagógico que sirva para la formación de estas comunidades indígenas y de su diversidad cultural tomando en cuenta su relación con un cúmulo educativo y la formación que emana la sociedad como miembros de un grupo o comunidad. Según (Rodríguez y Sanz, 1996a, pág. 23): "Todo esto en criterio de estos escritos, son además métodos de replanteamientos, de hipótesis y de muestras con diferente perspectiva que sirvan para el adiestramiento del colectivo en función de su formación cultural".

En relación con esto, (Flórez, 2008a, pág. 59): "un modelo es la imagen 
o representación del conjunto de relaciones que definen un fenómeno, con miras a su mejor entendimiento". En virtud de esto, se infiere a un acercamiento imaginario y adecuado a la representación y sabidurías conectadas con un fenómeno en particular, dado que la noción de un modelo es preciso instituir un estudio exclusivamente metódico donde no solamente esté reconocido en un todo sino también en un juicio de igualdad

Al respecto, (Flórez, 2008b, pág. 59): afirma que "los modelos pedagógicos representan formas particulares de interrelación entre los parámetros pedagógicos". Por esta razón, destaca la necesidad de utilizar procesos metódicos y didácticos los mismos sirven de guía para instruir sin condición a la comunidad. Bajo esta primicia el autor destaca algunos fundamentos tales como: instruir a la persona de manera correcta, tomando en cuenta sus habilidades y técnicas, así como: Programas y prácticas pedagógicas concretas, qué ritmo o niveles debe llevarse el proceso instructivo, quién administra este proceso educativo y en quien se ajusta.

Sobre la base de las ideas expuestas: este patrón cotidiano tuvo influencia en los métodos de instrucción y en las técnicas pedagógicas, esta visión influyó en la filosofía de grandes hombres como Aristóteles, en donde la enseñanza era fundamental para la ideología, también para el efecto y rescate de la renovación (Canfux, 1996, pág. 15).

En lo esencial, se puede afirmar que la pedagogía plantea una guía formativa habitual ajustada a la sensibilidad de un ideal creyente donde resalte el dictamen de cada perfil. Debe existir una analogía entre la persona sabiadiscípulo donde no solo se trasmite lo axiológico sino lo epistémico. Por lo tanto, el progreso de la humanidad se obtiene con la instrucción del perfil y de las jurisdicciones del alma.

El estándar conductista supone que la escuela es una institución encargada de transferir episteme que sea reconocido por la sociedad, según este estándar, la enseñanza es el efecto de los cambios más o menos 
permanentes de conducta y en consecuencia la instrucción es cambiada por las circunstancias del entorno. Al respecto, (Flórez, 2008c, pág. 31): expresa "esta guía se desenvolvió comparablemente de manera progresiva y financiera de los recursos en la etapa superior del individualismo, bajo el propósito minucioso del comportamiento beneficioso de la humanidad". El modelo ha sido idóneo y empírico en el sentido en que se toma como esencia la enseñanza, el estudio del comportamiento desde diferentes entornos tomado en cuenta la reflexión, la operacionalización, exactitud y observación.

De acuerdo con este patrón se busca la dirección, el comportamiento del ser dirigido y beneficioso en correspondencia a las reglas establecidas por el organismo. El docente desempeña su oficio y proyecta escenarios de enseñanza y de sus inducciones para obtener el comportamiento deseado, se adiestra para el propósito de enseñanza claramente determinado.

Un modelo progresista se basa en un pensamiento positivista donde se proyecta una teoría, fundamentada en doctrinas pedagógicas las mismas se conciben indiscutibles en las propuestas planteadas hacia la escuela que queremos, siendo el discípulo la formación del nuevo ser. Como se ha podido observar la escuela es una representación fundada desde la existencia e instrucción del ser humano y de esta manera cristianizar en cada lugar donde el niño se desenvuelve para su desarrollo evolutivo de vida.

(Rodríguez y Sanz, 1996b, pág. 20): "la escuela nueva resaltó el papel activo que debe tener el estudiante, transformó las funciones que debe asumir el profesor en el proceso educativo y mostró la necesidad y posibilidad de cambios en el desarrollo del mismo". El proceso formativo plantea que con la cultura social, la humanidad afirma su adecuado progreso. La nueva transformación educativa estableció la enseñanza con nuevas técnicas y perfeccionamiento del ser humano y su evolución tiene las más significativas alegorías.

Por su parte, (De Zubiria, 1994, pág. 11): "La escuela nueva rompe el 
paradigma tradicional que explicaba el aprendizaje como proceso de impresiones que desde el exterior se incrustan en el alumno. En su lugar, la nueva escuela defenderá la acción como condición y garantía del aprendizaje". Este escritor facilita algunas proposiciones primordiales para la escuela de hoy: su objetivo se basa en que la enseñanza no puede estar restringida, se debe preparar al individuo no para el momento sino para su existencia. Lo implícito formativo debe ajustarse de lo general a lo particular tomando en cuenta el aprendizaje del niño, donde él construya su propio conocimiento a través de experiencias propias.

Por lo tanto, este modelo se podría llamar propiamente una teoría del conocimiento y no del aprendizaje ni de la enseñanza, considerando que los seres humanos utilizan procesos cognitivos que son diferentes en los niños y en los adultos. Por lo tanto, se explica la instrucción como la afirmación de los métodos epistemológicos acontecidos en la enseñanza.

La propuesta de este patrón didáctico, destaca el valor de la práctica en el progreso de los métodos epistemológicos. En este aspecto, su participación incide en su perfil enérgico de su conocimiento y de su progreso epistémico tomando en cuenta las afirmaciones de algunas teorías de aprendizaje, resaltando tres fundamentos entre los cuales se destacan: - Un mecanismo hipotético que brinda herramientas precisas para valorar la evolución epistémica y honesta de las personas; - Como un instrumento ventajoso en la proyección de contenidos pedagógicos de programas educativos que contribuyan al diseño educativo como convenio y el progreso logrado por los niños; - Y en la desaparición de algunas técnicas de educación como la enseñanza por innovación.

Por lo tanto, la Pedagogía critica no solo se basa en presentar no solamente una dicho de diatriba, sino también en una expresión de perspectivas. Los maestros que usan dicha enseñanza en conglomerado con sus alumnos en la coparticipan con sus estudiantes en la meditación crítica de 
sus correctas dogmas. De este modo deliberan críticamente los "textos" que se manejan en las técnicas de aprendizaje, concibiendo por contenido no sólo los textos, sino también teorías únicas y, el conocimiento, que explican un habituado lenguaje.

Por su parte, el fin importante de una institución es proteger a sus estudiantes para que estén preparados de hallar su bienestar social y es por eso que se plantea un modelo al de los planteles cotidianos, en las que según las teorías de esta guía comienza un ambiente de recelo. Infundir en los niños nociones generosas antes de que sean personas idóneas de parecerlo sólo crea sujetos farsantes y temerosos, pues por medio de la desconfianza se aspira exigir el beneficio de alguno. Por esta razón para sentirse una persona realizada, feliz, autentica, requiere ser emancipado para elegir su apropiado camino. Indaga

Según este artículo, se busca la verdad y autonomía del niño para su progreso original, abierto e independiente. Los programas no están construidos anticipadamente, solo que se implementan a medida que el estudiante los requiere. Una característica primordial de esta disposición es que se plantea un estilo basado la cual busca una modificación del método pedagógico, de modo que el estudiante innove a su alrededor. En lo esencial (Flórez, 2008d, pág. 38): "la escuela es ajustada para su existencia, donde el estudiante innove, construya y experimente para desenvolverse en la sociedad".

También las investigaciones intervinieron eficazmente en las reflexiones ideológicas y educativas en América y sus arduas opiniones son elementos considerables hoy día. La Educación conjunta se basa en el análisis de las organizaciones de la sociedad que están direccionadas en la actualidad y en ejecución de la autoridad. Siguiendo el esquema, intercede en la evolución de la capacidad del pensamiento analítico con el fin de cambiar la humanidad. 
Así mismo, se pretende adquirir un compromiso provechoso e instructivo, por la integración de esta a todos los educandos equitativamente, en una formación vinculada, promoviendo un amplio repertorio lingüístico. Todo maestro que labora con este patrón, participa con sus alumnos en la meditación analítica de sus adecuados credos y prudencias. Igualmente disputan fuertemente las referencias de indagación, las cuales se emplean en la formación, dejando claro que las bases no solo son de los libros, sino también de los libros vivientes de la comunidad, las costumbres del entorno, la diversidad de argumentación que expresa un acontecimiento y su lengua conjunta.

Al igual, (Flórez, 2008e, pág. 33): manifiesta "que este patrón escudriña el progreso de las destrezas de las corrientes críticas que admiten al alumno participar enérgicamente en los cambios de la humanidad". Incita al análisis de la epistemología de la ciencia y su entorno constantemente. Se direcciona en la instrucción conglomerada y analítica de lo autónomo en sus costumbres y tradiciones. Este elemento educativo ocupa los entornos los cuales sirven de base para que los educandos realicen sus trabajos mancomunados y solucionen colectivamente las dificultades que no deben resolverse personalmente.

En este modelo, se realiza un entorno agradable, que proporcione al estudiante su dirección a las organizaciones epistémicas, el fin de este modelo es alcanzar que el estudiante tenga su prosecución de manera asertiva de acuerdo con su intelectualidad. El estudiante hará sus programas de instrucción, el docente será la guía de las prácticas fundamentada por intereses en cada individuo que le permita desarrollar su intelecto teniendo en cuenta sus intereses.

Del mismo modo, el docente establece un entorno alentador de costumbres el cual permitirá al individuo el camino a las organizaciones epistémicas en prosecución. Así mismo se pretende fortalecer el pensamiento 
de los estudiantes de manera que puedan construir su aprendizaje a través de experiencias propias.

El modelo integral tiene como propósito instruir el intelecto, habilidades y destrezas del educando en donde el conocimiento obedece a las técnicas y objetivos de cada etapa en ejecución y diferencias personales del alumno. Su exigencia se establece en el progreso de sus habilidades y motivaciones del estudiante.

El progreso se define en la humanidad, para un trabajo colectivo y productivo donde la educación juega un papel importante y ambos deben ir de la mano respaldando la amplitud de preparación conjunta, así como también variando el conocimiento en las innovaciones del sistema educativo.

\section{Metodología}

Metodológicamente fue una investigación de naturaleza descriptiva interpretativa donde se detalla todo lo observado en el recorrido de la investigación. Para (Rodríguez y García, 1999, pág. 44): "con esta metodología se indaga, observa, analiza, explica y se interpreta la cultura de un pueblo o grupo o tribu".

\section{Procedimiento de la Investigación}

La investigación se realizará en dos etapas:

1). Es descriptiva-interpretativa con base en la investigación etnográfica; y la 2). Es la de teorización o de construcción teórica.

A continuación, se presentan los cuatro objetivos específicos:

1). Develar los valores educativos y culturales de la mancomunidad indígena Timotes; 2). Indagar su trascurso de la enseñanza en su accionar habitual; 3). Identificar aspectos educativos de la etnia que se han extinguido a raíz del proceso de interculturalidad; y 4). Integrar en el modelo pedagógico 
los elementos encontrados en los representantes de la Mancomunidad Indígena.

En correspondencia con los objetivos referidos se aplicará el método etnográfico. Se asume el método etnográfico dado que es el que permite aprehender la forma de su existencia de un grupo social.

Esta investigación se centra en descubrir lo que acontece cotidianamente en los escenarios naturales donde ocurren los fenómenos educativos. En tal sentido, cuando se realiza etnografía se busca edificar un compendio imaginario que acumule y refleje todo lo cercano a las interpretaciones en cada una de sus faenas diarias. Bajo este caso específico se pretende describir el estándar de la transmisión y cultura étnica en el seno de la mancomunidad indígena El Paramito.

La selección del método está condicionada por la naturaleza del objeto de estudio y por las formas particulares de desarrollo que asume la realidad concreta a investigar. En ese aspecto, y dado que la naturaleza de la investigación involucra un grupo humano con su propia cultura en la mancomunidad indígena El Paramito, se entiende que el objeto de estudio se caracteriza por una configuración que integra en un todo coherente símbolos, valores, normas, creencias y actitudes de los actores involucrados.

En concordancia con lo anteriormente expuesto, la etnografía constituye la senda por la que se estudia la forma de subsistencia de un grupo en general pues (Rodríguez y Marcelo, 1999, pág. 44): manifiesta "la descripción o reconstrucción analítica de carácter interpretativo de la cultura, formas de vida y estructura social del grupo investigado". En ese orden de ideas, se pretende edificar un esbozo imaginario que acumule y reconozca verdaderamente las apreciaciones, tareas y patrones de dicho grupo étnico, esbozo constituido en este caso por un conjunto de parámetros de la práctica educativa en el seno de una etnia indígena, aún en el caso de no ser explícitos 
o aplicados de manera consciente.

\section{Resultados}

El proceso formativo pretende formar un indígena Timotes, como ser originario, orgulloso de su identidad, que recuerde sus raíces de grupo étnico glorioso de la historia del páramo andino. La persistencia de esta tribu hace pensar que existe un modo de específico de enseñar y transmitir de generación en generación sus conocimientos, costumbres y tradiciones entre las cuales se menciona:

\subsection{Método y experiencias de enseñanza}

El método de enseñanza se soporta fundamentalmente en tres formas: la oralidad, la imitación y el aprender haciendo.

\subsubsection{Oralidad}

Se refleja en el sistema de comunicación, está representada en la interacción de sus miembros, cuentos, canciones, mitos, juegos, diversiones, diálogos, narraciones históricas, para la familia y comunidad, cuyos depositarios son los ancianos. Es decir, enseñar cómo se hacen las cosas, en otras palabras, es la base de la sabiduría a través de tradiciones y representaciones de generación en generación. Además, la enseñanza en la trasmisión oral se manifiesta en el papel de los ancianos y la manera de enseñar, que se transmite boca en boca, la cual ejerce un rol fundamental como herencia cultural para la educación de las nuevas generaciones aborígenes.

\subsubsection{Imitación}

Es imitar lo que hacen los adultos para aprender, los ancianos educan 
para mantener lo que les pertenece por medio de lo que hacen. Hablando y enseñando como se hacían las cosas. Los más pequeños adquirían toda la cultura basada en un modelo de observación e imitación de la rutina diaria. El desenvolvimiento, el contacto directo y las acciones de los niños con los adultos esta relacionados con la enseñanza de la cultura, costumbres y tradiciones y hasta la actualidad aún persisten.

\subsubsection{Aprender haciendo}

Expresa en los más pequeños un sistema de saber propio. Hacer lo que los adultos hacen desde que el niño comienza a socializarse en la comunidad. Implica un proceso de formación intercultural como pueblo indígena, donde los más pequeños expresen su sistema de saberes propios, a través de su faena diaria los niños en el campo y las niñas en tareas domésticas imitan lo que los adultos hacen como la cultura ritos, juegos, canciones y otras acciones diarias.

\subsubsection{Trabajo-faena diaria}

Esta actividad forma parte de la construcción y reproducción de su identidad como pueblo. Es el medio de formación primordial de sus habitantes. Desde pequeños inician la faena del campo imitan desde edades tempranas el proceso de los cultivos, siembra, y recolección de rubros agrícolas, en la labor doméstica, las niñas aprenden todo lo relacionado al hogar en la figura materna. El Trabajo representa una tarea instructiva, socializadora y divertida, beneficioso para obtener producción. Es significar orgullo y la dignidad para el ser humano.

\subsubsection{Gastronomía Típica}

La herencia culinaria típica en trasmitida desde tiempos remotos como contribución de su acervo cultural. La gastronomía es el resultado de prácticas tradiciones y costumbres de las familias pertenecientes a la etnia. Para la 
preparación de los platos típicos los alimentos son autóctonos, los cuales le dan mayor fuerza a la cultura y representa el emblema primordial principales hechos que acontecen en las existencias de los indígenas y que aún se mantienen.

\subsubsection{Medicina Tradicional}

La comunidad indígena desarrolla de un conjunto de conocimientos, prácticas y tradiciones sobre el cuerpo humano. Para la mancomunidad Indígena la medicina tradicional representa la variedad de remedios y tratamientos para preservar y promover la salud, que pasan de generación en generación. Sus prácticas forman parte de la tradición oral, en su entorno y el contacto con la naturaleza.

\section{Análisis e interpretación de los resultados}

Con relación a los resultados, el saber colectivo entre los miembros de la comunidad es el espacio idóneo para el compartir de conocimientos, el cual contribuye a la formación en todas sus etapas de la vida. En la comunidad se viven experiencias cotidianas de su cultura lo que ha permitido que esta perdure en el tiempo que favorecen la transmisión de su cultura para que permanezca por más de doscientas años en este ámbito. La oralidad en la comunidad indígena objeto de investigación se concibe como un hecho comunicativo entre los miembros que conviven y viven su cultura como sistema primario de expresión y relaciones cotidianas; de hecho, su lenguaje es expresado a través de rasgos particulares que les da identidad propia.

Aunado a esta labor también se hizo evidente durante la observación de las actividades, puesto que ellas prevalecen las palabras y orientaciones de los adultos en la educación de los niños respecto de sus costumbres y tradiciones. Por esta razón las comunidades indígenas heredan y desarrollan conocimientos los cuales son trasmitidos de generación en generación donde 
los ancianos y adultos, educan, enseñando sobre los ancestros y los distintos modos de vida para desenvolverse en la sociedad. En síntesis, la transmisión oral es la plataforma de aquellas culturas que no están escritas, por lo que constituye la base de las comunidades, entendiendo que cada lengua, además, establece matrices que le son únicas y diferentes a las demás, confiriendo su identidad.

En consecuencia, la educación en la Mancomunidad indígena El Paramito se sustenta en el imitar las acciones de los abuelos, padres y adultos; de esta forma se transmiten los conocimientos de generación en generación durante sus faenas cotidianas o momentos especiales como rituales, reuniones, encuentros indígenas, fiestas en la comunidad, sus modos de actuar, los principios, valores, trabajo, costumbres, tradiciones. Así, los más pequeños adquieren todo lo ancestral y es el medio de aprendizaje. La enseñanza de las costumbres en este pueblo indígena ocurre en un aprender haciendo, pues las experiencias vividas reproducen patrones conductuales que observan de sus allegados y comunidad. Aquí, los ritos juegan un rol importante porque fijan en el aprendiz conceptos y prácticas relacionados con mitos, creencias, valores, historia y cultura de las etnias, ya que todo es transmitido en forma oral y vivencial.

Por esta razón, el ser indígena Timotes dignifica sus raíces, cultura, historia, en busca de las mitológicas huellas del aborigen. Los indígenas Timotes, fue la principal tribu que residía en el centro del amplio territorio que abarca los estados Trujillo y Mérida, y la que mejor supo conservar sus tradiciones e idioma. Finalmente pertenecer a la etnia es formar parte de nuestra vida, con vivencias hechos llenos de historias fascinantes y esplendorosas que nos llena de orgullo por lo que nos identifica como gente generosa de la comarca Timotes. 


\section{Conclusiones}

El proceso de transmisión de su cultura se realiza de forma oral, por imitación y aprenden en el hacer cotidiano, acompañando a los adultos. Comienza en la interacción familiar. Esta oralidad se da a lo largo de toda la vida, su lenguaje es expresado a través de rasgos particulares que les da identidad propia. También imitan las acciones de los abuelos, padres y adultos; de esta forma se transmiten los conocimientos de generación en generación durante sus faenas cotidianas o momentos especiales como rituales, reuniones, encuentros indígenas, fiestas en la comunidad, sus modos de actuar, los principios, valores, trabajo, costumbres y tradiciones.

La reconstrucción del modelo pedagógico subyacente en la Mancomunidad Indígena El Paramito permitió precisar que el proceso de su formación está dirigido a formar un ser indígena Timotes, capaz de vivir su cultura y lograr que perdure con sus tradiciones, costumbres, ritos, creencias y supersticiones. Sin embargo, al caracterizarse por ser oral, imitativa y práctica, su eje lo constituyen los ancianos, quienes en la medida que desaparezcan también pueden desaparecer su cultura.

En consecuencia, es importante su divulgación, reflexión y valoración como prácticas que se pueden realizar en el ámbito de comunidades originarias que permita incentivar a los miembros de las diferentes etnias a valorar desde la comprensión, la situación de riesgo que viven los pueblos indígenas venezolanos, por las amenazas contra su cultura. También concienciarlos en su corresponsabilidad en la conservación de su lengua, cultura, costumbres, dogmas, y modos de vida, su hábitat, a fin de mantener la identidad y diversidad cultural como población indígena.

\section{Reflexiones Finales}

Es necesario fomentar y valorar las culturas volverlas forjadoras de participación de identidad y de diferencias individuales y grupales. Los grupos 
étnicos reconocen su complejidad cultural tomando en cuenta sus valores, raíces, emblemas, ritos, modos de vida, culto y leyenda, para vigorizar la identidad múltiple de un grupo en general. Esto significa que no se trata de posesionarse de una cultura sino de varias manteniendo sus raíces, naturaleza, dogmas, representaciones como parte de un territorio.

La Mancomunidad Indígena El Paramito radicada en Timotes, si bien ha mantenido su cultura a lo largo del tiempo, la problemática se presenta actualmente, que paulatinamente se está perdiendo la lengua originaria, su propia cultura, tradiciones, mitologías, costumbres, sus formas de organización mancomunada y sus conocimientos hereditarios ineludibles para la conservación y ciencia de su educación. En consecuencia, la presente investigación tuvo como propósito reconstruir el modelo pedagógico subyacente en el proceso de la educación étnica que practica dicha Mancomunidad.

Además de describir la intención de esta comunidad, se observa la enseñanza formativa en cada uno de sus integrantes de la mancomunidad indígena Timotes. Además de la cultura se observaron acciones en la forma como cada uno se comunica a través de su lenguaje y, como veneran la naturaleza como algo sagrado del todopoderoso, las mismas enseñanzas son transferidas por sus antepasados. Igualmente, la cultura de este grupo se conserva en cada uno de sus habitantes siendo su primordial rasgo la imitación y la oralidad.

\section{Referencias}

Bastidas, L. (2003). De los timoto-cuicas a la invisibilidad del indígena andino y a su diversidad cultural. Boletín Antropológico, 21(59), 283312, ISSN: 1325-2610. Recuperado de:

http://www.redalyc.org/articulo.oa?id=71205903

Canfux. V. (1996). La diversidad en la práctica educativa. Modelos de 
orientación y tutoría. Madrid, España: Editorial CCS, pág. 15.

De Zubiria, J. (1994). Tratado de Pedagogía Conceptual: Los modelos pedagógicos. Santafé de Bogotá, Colombia: Fundación Merani. Fondo de Publicaciones Bernardo Herrera Merino, pág. 11.

Flórez, O. (2008a,b,c,d,e). Hacia una pedagogía del conocimiento. Santafé de Bogotá, Colombia: McGraw-Hill, pág. 59.

Pérez, M. (2016). La Educación Intercultural. Revista Scientific, 1(2), 162180. Recuperado de: https://doi.org/10.29394/scientific.issn.25422987.2016.1.2.10.162-180

Rodríguez, A., \& Sanz, T. (1996a,b). La escuela nueva. La Habana, Cuba: Centro de estudios para el perfeccionamiento de la educación superior CEPES, pág. 23.

Rodríguez, D., \& Marcelo, A. (1999). Sociedad y teoría de sistemas. 3oe., ISBN: 956-11-1508-5. Santiago de Chile: Editorial Universitaria, El Saber y la Cultura, pág. 44.

Rodríguez, G., \& García, E. (1999). Metodología de la investigación cualitativa. $2^{\mathrm{a}}$ ed. España: Aljibe.

Yampara S. (2010). Estrategias didácticas y organizativas ante la diversidad: dilemas del profesorado. Barcelona, España: Octaedro. 


\section{María del Carmen Pérez Paredes}

e-mail: miduam@hotmail.com

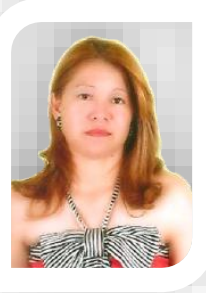

Nacida en Timotes, Estado Mérida, Venezuela. Título de Doctora en la Universidad Rafael María Baralt, en Valera, Estado Trujillo, Magister Scientiarum en Administración de la Educación Básica. Abogado de la República Bolivariana de Venezuela, Misión Sucre. Licenciada en Educación Básica integral en la Universidad Alonso de Ojeda, estado Zulia, Técnico Superior Universitario en Educación Integral, Instituto Universitario de Tecnología Mario Briseño Iragorry, Valera estado Trujillo. Ponente en congreso pedagógico municipal, jurado evaluador de tesis de grado en la Universidad Nacional Experimental de la Seguridad (UNES) y Universidad Nacional Rafael María Baralt. En la actualidad con una experiencia en aula de clase como docente en diferentes grados de primaria y educación media general, también a nivel Universitario (UNEFA), con dos años de experiencia. Coordinadora Pedagógica en el Núcleo Escolar Rural № 196 Estado Mérida desde el 2013 hasta la presente fecha. 